\title{
ON O. BONNET III-ISOMETRY OF SURFACES IN THREE DIMENSIONAL EUCLIDEAN SPACE
}

\author{
WENMAO YANG
}

(Received 23 January 1989)

Communicated by K. C. H. Mackenzie

\begin{abstract}
In this paper we consider O. Bonnet III-isometry (or BIII-isometry) of surfaces in 3-dimensional Euclidean space $E^{3}$. Suppose a map $F: M \rightarrow M^{*}$ is a diffeomorphism, and $F^{*}\left(\mathrm{III}^{*}\right)=\mathrm{III}$, $\kappa_{i}(m)=\kappa_{i}^{*}\left(m^{*}\right), i=1,2$, where $m \in M, m^{*} \in M^{*}, m^{*}=F(m), \kappa_{i}$ and $\kappa_{i}^{*}$ are the principal curvatures of surfaces $M$ and $M^{*}$ at the points $m$ and $m^{*}$, respectively, III and III $^{*}$ are the third fundamental forms of $M$ and $M^{*}$, respectively. In this case, we call $F$ an O. Bonnet III-isometry from $M$ to $M^{*}$. O. Bonnet I-isometries were considered in references [1]-[5].

We distinguish three cases about BIII-surfaces, which admits a non-trivial BIII-isometry. We obtain some geometric properties of BIII-surfaces and BIII-isometries in these three cases; see Theorems 1, 2, 3 (in Section 2). We study some special BIIl-surfaces: the minimal BIIIsurfaces; BIII-surfaces of revolution; and BIII-surfaces with constant Gaussian curvature; see Theorems 4, 5, 6 (in Section 3).
\end{abstract}

1980 Mathematics subject classification (Amer. Math. Soc.) (1985 Revision): 53 A 05, 53 B 20.

\section{Introduction}

O. Bonnet [1] was the first to study the isometric deformations of surfaces in 3-dimensional Euclidean space $E^{3}$ which preserve mean curvature. Also W. C. Graustein [4] and E. Cartan [2] did some work in this area. Recently, S. S. Chern [3] obtained an interesting result about the surfaces with mean curvature $H \neq$ constant. After that, I. M. Roussos [5] got some detailed results. In this paper, a more general definition of $O$. Bonnet deformations is given.

(C) 1990 Australian Mathematical Society 0263-6115/90\$A2.00+0.00 
Let $M$ and $M^{*}$ be two surfaces in the Euclidean space $E^{3}$. Suppose I, II, III are the first, second, third fundamental forms of the surface $M$, respectively. We shall denote the quantities pertaining to $M^{*}$ by the same symbols with asterisks " *".

Definition. Suppose $F: M \rightarrow M^{*}$ is a diffeomorphism, and $F^{*}\left(\mathrm{I}^{*}\right)=\mathrm{I}$ or $F^{*}\left(\mathrm{II}^{*}\right)=\mathrm{II}$ or $F^{*}\left(\mathrm{III}^{*}\right)=\mathrm{III}$, where $F^{*}$ represents $F$ 's cotangent map. Then we call $F$ a I- or II- or III-isometry of $M$ and $M^{*}$, respectively. Moreover, suppose $F$ preserves the principal curvatures at the corresponding points:

$$
\kappa_{i}(m)=\kappa_{i}^{*}\left(m^{*}\right), \quad m^{*}=F(m), i=1,2,
$$

where $m \in M, m^{*} \in M^{*}, \kappa_{i}$ and $\kappa_{i}^{*}$ are the principal curvatures of $M$ and $M^{*}$. In this case, we call $F$ an $O$. Bonnet I or II or III-isometry, denoted by BI or BII or BIII-isometry, respectively. If a surface $M$ admits a non-trivial BI or BII or BIII-isometry, we call $M$ a Bonnet I or II or III-surface, respectively.

Isometric deformations which were considered in [1]-[5] are BI-isometries, because an isometric deformation preserves Gaussian curvature $K$, so if the map preserves mean curvature $H$, then it preserves the two principal curvatures $\kappa_{1}$ and $\kappa_{2}$, and hence it is a BI-isometry.

In the present paper we shall study BIII-isometries and obtain some results given in Theorems 1-6, which are shown to be similar to the case of BIisometries.

\section{Lemmas and formulas}

We shall let $\omega=\omega_{1}+i \omega_{2}\left(i^{2}=-1\right)$ be the complex structure of the metric $I=\left(\omega_{1}\right)^{2}+\left(\omega_{2}\right)^{2}$ and let $\omega_{12}$ be the connection form associated to $\mathrm{I}$, which is determined by the structural equations

$$
d \omega_{1}=-\omega_{2} \wedge \omega_{12}, \quad d \omega_{2}=\omega_{1} \wedge \omega_{12}
$$

or

$$
d \omega=i \omega \wedge \omega_{12},
$$

where $\omega_{1}$ and $\omega_{2}$ are two real linearly independent forms.

Lemma 1. Suppose $\omega$ is a complex structure.

(i) If $\omega^{*}=\bar{\omega}$, then $\omega_{12}^{*}=-\omega_{12}$.

(ii) If $\omega^{*}=e^{i \tau} \omega$, then $\omega_{12}^{*}=\omega_{12}-d \tau$.

(iii) If $\omega^{*}=A \omega$, then $\omega_{12}^{*}=\omega_{12}+* d \log A$. 
Here $\tau, A$ are functions, and "*" is the Hodge *-operator with

$$
* \omega_{1}=\omega_{2}, \quad * \omega_{2}=-\omega_{1} .
$$

We consider a piece of an oriented surface $M$ in $E^{3}$, which we assume to be sufficiently differentiable and with no umbilic points and non-zero Gaussian curvature. Over $M$ there is a field of orthonormal frames $m e_{1} e_{2} e_{3}$, such that $m \in M$, where unit vectors $e_{1}$ and $e_{2}$ are the principal directions of $M$ at $m$, and $e_{3}$ is the unit normal vector to $M$ at $m$. Suppose $\omega_{1}$ and $\omega_{2}$ are a basis of the 1-forms of $M$ dual to the field of principal frames. Set $a>c$, and

$$
\begin{aligned}
\omega_{1} & =a \omega_{13}, \quad \omega_{2}=c \omega_{23}, \quad a c \neq 0, \\
\omega_{12} & =h \omega_{13}+k \omega_{23} .
\end{aligned}
$$

The mean curvature and the Gaussian curvature of $M$ are

$$
H=\frac{1}{2}\left(a^{-1}+c^{-1}\right), \quad K=(a c)^{-1} .
$$

The structural equations of $M$ are

$$
\begin{aligned}
d \omega_{1} & =-\omega_{2} \wedge \omega_{12}, \quad d \omega_{2}=\omega_{1} \wedge \omega_{12}, \\
d \omega_{12} & =-K \omega_{1} \wedge \omega_{2}=-\omega_{13} \wedge \omega_{23}, \\
d \omega_{13} & =-\omega_{23} \wedge \omega_{12}, \quad d \omega_{23}=\omega_{13} \wedge \omega_{12} .
\end{aligned}
$$

The metric of the Gaussian image $g(M)$ of $M$ is

$$
I_{g}=\left(\omega_{13}\right)^{2}+\left(\omega_{23}\right)^{2}
$$

The complex structure of this metric is

$$
\omega=\omega_{13}+i \omega_{23} \text {. }
$$

From (6), we have

$$
d \omega=i \omega \wedge \omega_{12}
$$

We denote

$$
f=a-c>0, \quad g=a+c=2 H K^{-1} .
$$

Taking exterior derivatives of (1) and using (4) and (6), we get the existence of functions $\alpha, \beta, \nu, \delta$, such that

$$
\begin{aligned}
d a & =\alpha \omega_{13}+\beta \omega_{23}=\alpha \omega_{13}+f h \omega_{23}, \\
f \omega_{12} & =\beta \omega_{13}+\nu \omega_{23}, \quad \beta=f h, \quad \nu=f k, \\
d c & =\nu \omega_{13}+\delta \omega_{23}=f k \omega_{13}+\delta \omega_{23} .
\end{aligned}
$$


Taking exterior derivatives of (11) and using (5), (6) and (11), we get the existence of $A, B, \ldots, E$, such that

$$
\begin{aligned}
d \alpha & =3 \beta \omega_{12}+A \omega_{13}+B \omega_{23}=\left(A+3 f h^{2}\right) \omega_{13}+(B+3 f h k) \omega_{23}, \\
d \beta & =-(\alpha+2 \nu) \omega_{12}+B \omega_{13}+(C+a) \omega_{23} \\
& =\left[B-f h\left(\alpha f^{-1}-2 k\right)\right] \omega_{13}+\left[C+a-f h\left(\alpha f^{-1}-2 k\right)\right] \omega_{23}, \\
d \nu & =(\delta+2 \beta) \omega_{12}+(C+c) \omega_{13}+D \omega_{23} \\
& =\left[C+c+f h\left(\delta f^{-1}-2 h\right)\right] \omega_{13}+\left[D+f k\left(\delta f^{-1}-2 h\right)\right] \omega_{23}, \\
d \delta & =-3 \nu \omega_{12}+D \omega_{13}+E \omega_{23}=(D-3 f h k) \omega_{13}+\left(E-3 f k^{2}\right) \omega_{23} .
\end{aligned}
$$

Using (10) and (11), we get

$$
\begin{aligned}
d g & =2 d\left(H K^{-1}\right)=f\left(u \omega_{13}+v \omega_{23}\right), \\
d f & =f\left[(u-2 k) \omega_{13}-(v-2 h) \omega_{23}\right],
\end{aligned}
$$

where

$$
f u=\alpha+\nu, \quad f v=\beta+\delta .
$$

Then we can determine the following 1-forms. By using $u, v$ in (15), let

$$
\begin{array}{ll}
\theta_{1}=u \omega_{13}+v \omega_{23}, & \theta_{2}=* \theta_{1}=-v \omega_{13}+u \omega_{23}, \\
\alpha_{1}=u \omega_{13}-v \omega_{23}, & \alpha_{2}=* \alpha_{1}=v \omega_{13}+u \omega_{23} .
\end{array}
$$

If $H K^{-1}=$ constant, then $\theta_{i}=\alpha_{i}=0$; if $H K^{-1} \neq$ constant, $\theta_{1}$ and $\theta_{2}$, or $\alpha_{1}$ and $\alpha_{2}$ are linearly independent. From (13) and (14), it follows that

$$
\begin{aligned}
& d g=f \theta_{1}, \\
& d \log f=\alpha_{1}+2 * \omega_{12} .
\end{aligned}
$$

According to (3),

$$
4 K^{-1}=g^{2}-f^{2} \text {. }
$$

Taking derivatives of (20) and using (18) and (19), we have

$$
2 f^{-1} d\left(K^{-1}\right)=g \theta_{1}-f\left(\alpha_{2}+2 * \omega_{12}\right) \text {. }
$$

Suppose $H K^{-1} \neq$ constant. We denote

$$
\begin{aligned}
& u+i v=L e^{i \psi}, \\
& L^{2}=u^{2}+v^{2}=f^{-2}\left[\alpha^{2}+\beta^{2}+\nu^{2}+\delta^{2}+2(\alpha \nu+\beta \delta)\right] . \\
& \cos \psi=u L^{-1}, \quad \sin \psi=v L^{-1} .
\end{aligned}
$$

Let

$$
\begin{aligned}
& \theta=\theta_{1}+i \theta_{2}, \\
& \alpha=\alpha_{1}+i \alpha_{2} .
\end{aligned}
$$


Using (8), (16), (17), and (22), we get

$$
\theta=L e^{-i \psi} \omega, \quad \alpha=L e^{i \psi} \omega, \quad \theta=e^{-2 i \psi} \alpha,
$$

$$
\theta_{1}=\alpha_{1} \cos 2 \psi+\alpha_{2} \sin 2 \psi=L\left(\omega_{13} \cos \psi+\omega_{23} \sin \psi\right) \text {, }
$$

(27) $)^{\prime} \quad \theta_{2}=-\alpha_{1} \sin 2 \psi+\alpha_{2} \cos 2 \psi=L\left(-\omega_{13} \sin \psi+\omega_{23} \cos \psi\right)$, $\alpha_{1}=L\left(\omega_{13} \cos \psi-\omega_{23} \sin \psi\right), \quad \alpha_{2}=L\left(\omega_{13} \sin \psi+\omega_{23} \cos \psi\right)$.

From (10), (20), (23) and (13), it follows that

$$
f^{2}=4\left[\left(H K^{-1}\right)^{2}-K^{-1}\right], \quad[\operatorname{grad}(g)]^{2}=4\left[\operatorname{grad}\left(H K^{-1}\right)\right]^{2}=f^{2} L^{2},
$$

so

$$
L^{2}=4 f^{-2}[\operatorname{grad}(g)]^{2}=\frac{\left[\operatorname{grad}\left(H K^{-1}\right)\right]^{2}}{\left(H K^{-1}\right)^{2}-K^{-1}} .
$$

We now consider a metric which is conformal to $I_{g}$ (see (7))

$$
\widehat{I}=\left(\alpha_{1}\right)^{2}+\left(\alpha_{2}\right)^{2}=L^{2} I_{g} .
$$

Let $\theta_{12}$ and $\alpha_{12}$ be the connection forms associated to complex structures $\theta$ and $\alpha$, respectively. From (27), using Lemma 1, we have

$$
\begin{aligned}
& \theta_{12}=\omega_{12}+d \psi+* d \log L, \\
& \alpha_{12}=\omega_{12}-d \psi+* d \log L, \\
& \theta_{12}=\alpha_{12}+2 d \psi .
\end{aligned}
$$

We rewrite (2) as

$$
\omega_{12}=h^{\prime} \alpha_{1}+k^{\prime} \alpha_{2}
$$

From $(2)^{\prime}$ and (27), we have

$$
\begin{aligned}
& f L h^{\prime}=f^{-1}(h \cos \psi-k \sin \psi)=\alpha \beta-\nu \delta, \\
& f L k^{\prime}=f^{-1}(h \sin \psi+k \cos \psi)=\alpha \nu+\beta \delta+\beta^{2}+\nu^{2} .
\end{aligned}
$$

Taking derivatives of (23) and using (11), (12), (18) and (19), we get

$$
d \log L=-\alpha_{1}-2 * \omega_{12}+* \Omega+\rho \theta_{1},
$$

where

$$
\begin{aligned}
& 2 f L^{2} \Omega=2(B+D) \alpha_{1}-(A-E-f) \alpha_{2}, \\
& 2 f L^{2} \rho=A+2 C+E+2 H K^{-1} .
\end{aligned}
$$

From (24), it can be seen that

$$
u \sin \psi-v \cos \psi=0 .
$$


Taking derivatives of $(24)^{\prime}$, using (11) and previous formulas, we get

$$
d \psi=-\omega_{12}+\Omega+\rho \theta_{2} .
$$

Inserting (34) and (37) into (30) and (31), we get

$$
\begin{aligned}
& \theta_{12}=2 \omega_{12}-\alpha_{2}+2 \rho \theta_{2}, \\
& \alpha_{12}=4 \omega_{12}-\alpha_{2}-2 \Omega=2 \omega_{12}-\alpha_{2}-2 d \psi+2 \rho \theta_{2} .
\end{aligned}
$$

Let

$$
\alpha_{12}=P \alpha_{1}+Q \alpha_{2},
$$

where $P, Q$ are two functions. Using (39), (35), (36), (2) ${ }^{\prime}$ and (33) gives

$$
\begin{aligned}
& P=-2(f L)^{-2}[f(B+D)+2(\alpha \beta-\gamma \delta)], \\
& Q=1+(f L)^{-2}\left[f(A-E-f)-2\left(\alpha^{2}-\beta^{2}-\nu^{2}+\delta^{2}\right)\right] .
\end{aligned}
$$

By solving (39), we have

$$
\Omega=2 \omega_{12}-\frac{1}{2}\left[P \alpha_{1}+(Q+1) \alpha_{2}\right] .
$$

Inserting (39) $)^{\prime}$ into (34) and (37), we get

$$
\begin{aligned}
& 2 d \log L=(Q-1) \alpha_{1}-P \alpha_{2}+2 \rho \theta_{1}, \\
& 2 d \psi=2 \omega_{12}-\left[P \alpha_{1}+(Q+1) \alpha_{2}\right]+2 \rho \theta_{2} .
\end{aligned}
$$

Set

$$
d P=P_{i} \alpha_{i}, \quad d Q=Q_{i} \alpha_{i}, \quad d \rho=\rho_{i} \alpha_{i}, \quad i=1,2 .
$$

Taking exterior derivatives of (42), we have

$$
\left(d \rho-\rho * \theta_{12}\right) \wedge \theta_{1}+J \theta_{1} \wedge \theta_{2}=0,
$$

where

$$
-2 J=P+P_{1}+Q_{2} .
$$

Taking exterior derivatives of (43), we have

$$
\left(d \rho-\rho * \theta_{12}\right) \wedge \theta_{2}-I \theta_{1} \wedge \theta_{2}=0,
$$

where

$$
2 I=\widehat{K}-Q-2 L^{-2}
$$

and $\widehat{K}$ is the Gaussian curvature of the metric $\widehat{I}$ (see (29)) so that

$$
d \alpha_{12}=-\widehat{K} \alpha_{1} \wedge \alpha_{2}, \quad \widehat{K}=-P^{2}-Q^{2}+P^{2}-Q_{1} .
$$

From (45) and (47), we obtain

$$
d \rho-\rho * \theta_{12}=-I \theta_{1}+J \theta_{2}
$$


or, by (32) and (48),

$$
\begin{aligned}
d \rho & =\rho * \theta_{12}-I \theta_{1}+J \theta_{2} \\
& =\rho\left(* \alpha_{12}+2 * d \psi\right)+\left[L^{-2}-\frac{1}{2}(\widehat{K}-Q)\right] \theta_{1}+J \theta_{2} .
\end{aligned}
$$

Taking exterior derivatives of [19] and using (40), we get

$$
d * \omega_{12}=-\frac{1}{2} P \alpha_{1} \wedge \alpha_{2} .
$$

Applying the *-operator and taking exterior derivatives of (40), we get

$$
d * \alpha_{12}=\left(P_{1}+Q_{2}\right) \alpha_{1} \wedge \alpha_{2} .
$$

Similarly, from (31), using (51) and (52), we get

$$
d * d \psi=\left(2 J+\frac{1}{2} P\right) \alpha_{1} \wedge \alpha_{2}
$$

and from (30) and (19), we have

$$
\begin{gathered}
d * \theta_{12}=2 J \alpha_{1} \wedge \alpha_{2}, \\
d * d \log f=\left(Q+2 L^{-2}\right) \alpha_{1} \wedge \alpha_{2} .
\end{gathered}
$$

Applying the *-operator to (34) and using (37), we get

$$
* d \log L=-\omega_{12}+\alpha_{12}+d \psi \text {. }
$$

Taking exterior derivatives of the above equation,

$$
d * d \log L=\left(L^{-2}-\widehat{K}\right) \alpha_{1} \wedge \alpha_{2} .
$$

We denote

$$
d \psi=\psi_{i} \alpha_{i}, \quad d J=J_{i} \alpha_{i}, \quad d(\widehat{K}-Q)=(\widehat{K}-Q)_{i} \alpha_{i}, \quad i=1,2 .
$$

Taking exterior derivatives of $(50)$ gives

$$
\begin{gathered}
\left(-I \theta_{1}+J \theta_{2}\right) \wedge * \theta_{12}+\left(I \theta_{2}+J \theta_{1}\right) \wedge \theta_{12}+I\left(d * \alpha_{12}+2 d * d \psi\right) \\
-\left[\frac{1}{2} d(\widehat{K}-Q)-d L^{-2}\right] \wedge \theta_{1}+d J \wedge \theta_{2}=0 .
\end{gathered}
$$

Let us compute the left side in (59).

(a) The sum of the first two terms is

$$
\begin{aligned}
& \left(-I \theta_{1}+J \theta_{2}\right) \wedge\left(* \alpha_{12}+2 * d \psi\right)+\left(I \theta_{2}+J \theta_{1}\right) \wedge \theta_{12} \\
& =\left\{\left[-I\left(P+1 \psi_{1}\right)+J\left(Q+2 \psi_{2}\right)\right] \cos 2 \psi\right. \\
& \left.-\left[I\left(Q+2 \psi_{2}\right)+J\left(P+2 \psi_{1}\right)\right] \sin 2 \psi\right\} \alpha_{1} \wedge \alpha_{2} .
\end{aligned}
$$

(b) The third term is

$$
I\left(d * \alpha_{12}+2 d * d \psi\right)=2 \rho J \alpha_{1} \wedge \alpha_{2} .
$$


(c) The sum of the last two terms is

(62)

$$
\begin{aligned}
& -\left[\frac{1}{2} d(\widehat{K}-Q)-d L^{-2}\right] \wedge \theta_{1}+d J \wedge \theta_{2} \\
& =-2 L^{-2} d \log L \wedge \theta_{1}-\frac{1}{2} d(\widehat{K}-Q) \wedge \theta_{1}+d J \wedge \theta_{2} \\
& =\left\{\left[\frac{1}{2}(\widehat{K}-Q)_{2}+J_{1}\right] \cos 2 \psi-\left[\frac{1}{2}(\widehat{K}-Q)_{1}-J_{2}\right] \sin 2 \psi\right. \\
& \left.\quad-L^{-2}[P \cos 2 \psi+(Q-1) \sin 2 \psi]\right\} \alpha_{1} \wedge \alpha_{2} .
\end{aligned}
$$

Inserting (60)-(62) into (59), we get

(63) $\rho J-\frac{1}{2} L^{-2}\left[P \cos 2 \psi_{+}(Q+1) \sin 2 \psi\right]$

$$
\begin{aligned}
& +\left\{-I\left(P+2 \psi_{1}\right)+J\left(Q+2 \psi_{2}\right)+\frac{1}{2}\left[\frac{1}{2}(\widehat{K}-Q)_{2}+J_{1}\right]\right\} \cos 2 \psi \\
& -\left\{J\left(P+2 \psi_{1}\right)+I\left(Q+2 \psi_{2}\right)+\frac{1}{2}\left[\frac{1}{2}(\widehat{K}-Q)_{1}-J_{2}\right]\right\} \sin 2 \psi=0 .
\end{aligned}
$$

We need the following lemma.

LEMMA 2. A necessary and sufficient condition for a surface $M$ with $H K^{-1}$ $\neq$ constant to be a Weingarten-surface is

$$
\left(P+2 \psi_{1}\right) \cos 2 \psi+\left(Q+2 \psi_{2}\right) \sin 2 \psi=0
$$

Proof. According to (10), (18) and (21), a necessary and sufficient condition for $M$ to be a $W$-surface is $d a \wedge d c=0$, which can be written as

$$
\left(\alpha_{1}+2 * \omega_{12}\right) \wedge \theta_{1}=0
$$

Applying the *-operator to (39), we get

$$
\alpha_{1}+2 * \omega_{12}=* \alpha_{12}+2 * d \psi+2 \rho \theta_{1} \text {. }
$$

Using the above equation from (65), we have

$$
*\left(\alpha_{12}+2 d \psi\right) \wedge \theta_{1}=0
$$

or

$$
\left(\alpha_{12}+2 d \psi\right) \wedge \theta_{2}=0 \text {. }
$$

Using (40), (58), (27) $)^{\prime}$ and rewriting the above equation, we see that (64) follows.

\section{BIII-isometry}

Let $F: M \rightarrow M^{*}$ be a III-isometry from $M$ to $\stackrel{*}{M}$, with $m e_{1} e_{2} e_{3}$ and $\dot{m}_{1}^{*} \dot{e}_{2} \ddot{e}_{3}$ the fields of principal frames over $M$ and $\stackrel{*}{M}$, respectively. We have

(1) $\omega_{1}=a \omega_{13}, \quad \omega_{2}=c \omega_{23}, \quad \stackrel{*}{\omega}{ }_{1}=\stackrel{*}{a} \stackrel{*}{\omega}_{13}, \quad \stackrel{*}{\omega}_{2}=\stackrel{*}{c} \stackrel{*}{\omega}_{23}$. 
Let

$$
\omega=\omega_{13}+i \omega_{23}, \quad \stackrel{*}{\omega}=\stackrel{*}{\omega}_{13}+i \stackrel{*}{\omega}_{23} .
$$

Since $F$ is a III-isometry, we have

$$
\stackrel{*}{\omega}=e^{i \tau} \omega
$$

or

$(2)^{\prime} \quad \stackrel{*}{\omega}_{13}=\omega_{13} \cos \tau-\omega_{23} \sin \tau, \quad \stackrel{*}{\omega}_{23}=\omega_{13} \sin \tau+\omega_{23} \cos \tau$,

where $\tau$ is an angle of rotation of the principal directions during the BIIIisometric deformation. On the other hand, from the invariance of principal curvatures, we get

$$
\stackrel{*}{a}=a, \quad \stackrel{*}{c}=c .
$$

Using (1.10) and (3) gives

$$
\stackrel{*}{f}=f, \quad \stackrel{*}{g}=g .
$$

From (1.16), (1.33) and (1.24),

$$
\stackrel{*}{\theta}_{1}=\theta_{1}
$$

or

$$
\stackrel{*}{u} \stackrel{*}{\omega}_{13}+\stackrel{*}{v} \stackrel{*}{\omega}_{23}=u \omega_{13}+v \omega_{23},
$$

which gives, in view of $(2)^{\prime}$,

$$
\stackrel{*}{u}=u \cos \tau-v \sin \tau, \quad \stackrel{*}{v}=u \sin \tau+v \cos \tau .
$$

Taking derivatives of the first equation in (4) and using (1.19) we get

$$
\stackrel{*}{\alpha}_{1}+2 * \stackrel{*}{\omega}_{12}=\alpha_{1}+2 * \omega_{12} .
$$

Using Lemma 1 from (2), we have

$$
\stackrel{*}{\omega}_{12}=\omega_{12}-\tau
$$

Using (7), (8), we have

$$
d \tau=\frac{1}{2}\left(\alpha_{2}-\stackrel{*}{\alpha}_{2}\right)
$$

From (6) and (1.27)'

$$
\stackrel{*}{\alpha_{2}}=\alpha_{1} \sin 2 \tau+\alpha_{2} \cos 2 \tau
$$

Putting

$$
t=\operatorname{ctg} \tau,
$$


we get from (9),

$$
d t=t \alpha_{1}-\alpha_{2} .
$$

This is the total differential equation satisfied by the angle $\tau$. In order that the BIII-isometry be non-trivial it is both necessary and sufficient that (12) is integrable. Taking exterior derivatives of (12), in view of (1.40), we get an integrable condition

$$
t P+1-Q=0 .
$$

Now let us distinguish three cases about BIII-isometry. Similarly, BIisometry is classified into three types.

(1) First type, $H K^{-1}=$ constant. Then by (1.13), $\alpha_{i}=0, i=1,2$.

(2) Second type, $H K^{-1} \neq$ constant, and $P \equiv 0, Q \equiv 1$. Then (13) holds identically for all $t$, and (12) has a continuum of solutions, each depending on an arbitrary constant. Thus we obtain a one-parameter family of surfaces BIII-isometric to $M$.

(3) Third type, $H K^{-1} \neq$ constant, and $P \not \equiv 0, Q \not \equiv 1$. Then from (13), we have

$$
t=(Q-1) P^{-1},
$$

and (12) has a single solution. Thus we obtain a single surface which is BIII-isometric to $M$.

THEOREM 1. Any surface with constant $\mathrm{HK}^{-1}$ is a BIII-surface of the first type. In other words, any surface with constant $\mathrm{HK}^{-1}$ can be III-isometrically deformed, preserving the principal curvatures. During this deformation the principal directions are rotated by a fxed angle $\tau$ (=constant).

Since in this case $\alpha_{1}=\alpha_{2}=0, d t=0, t=$ constant, $\tau=$ constant, Theorem 1 naturally holds. This theorem is an analogy of $O$. Bonnet's theorem for BI-isometries [1].

Theorem 2. Let $M$ be a BIII-surface of the second type, that is, $H K^{-1} \neq$ constant and $P \equiv 0, Q \equiv 1$.

(i) The metric which is conformal to the metric $I_{g}$ of the Gaussian image $g(M)$ of $M$,

$$
\widehat{I}=\frac{\left[\operatorname{grad}\left(H K^{-1}\right)\right]^{2}}{\left(H K^{-1}\right)^{2}-K^{-1}} I_{g},
$$

has Gaussian curvature equal to -1 , where $H$ and $K$ are the mean curvature and Gaussian curvature of $M$, respectively.

(ii) $M$ is $a W$-surface. 
(iii) The non-trivial family of BIII-surfaces is a family of surfaces which depends on six arbitrary constants.

Proof. Since

$$
P \equiv 0, \quad Q \equiv 1,
$$

(1.3) is identically true for all $t$. Using (1.49), we have

$$
\widehat{K}=-\left(P^{2}+Q^{2}-P_{2}+Q_{1}\right)=-1 .
$$

From (14), $P_{i}=Q_{i}=0$ and using (1.46) and (1.48), we get

$$
J=0, \quad I=-\left(1+L^{-2}\right), \quad J_{i}=0, \quad \widehat{K}_{i}=0 .
$$

Inserting the above equations into (1.63) we get

$$
2 \psi_{1} \cos 2 \psi+\left(1+2 \psi_{2}\right) \sin 2 \psi=0 .
$$

This is exactly (1.64). By Lemma 2 , we obtain (ii).

From (15) we have

$$
2 \psi_{1}=p \sin 2 \psi, \quad 1+2 \psi_{2}=-p \cos 2 \psi,
$$

where $p$ is a function. Taking derivatives of (16), we get, for $i=1,2$,

$$
2 \psi_{1 i}=p_{i} \sin 2 \psi+2 p \psi_{i} \cos 2 \psi, \quad 2 \psi_{2 i}=p_{i} \cos 2 \psi+2 p \psi_{i} \sin 2 \psi .
$$

Inserting (17) into (1.53), using $\psi_{12}=\psi_{21}$ plus $J=0, P=0$, and by solving the equation obtained, we get

$$
p_{1}=-2 p \psi_{2}, \quad p_{2}=2 p \psi_{1} .
$$

It can be verified by differentiating (18) that the integrable condition for $p$ is satisfied. From our discussion the differentials of the six functions $a, c$, $\log L, \rho, \psi, p$ are all determined. Hence our surfaces of non-constant $H K^{-1}$, which can be III-isometrically deformed in a non-trivial way preserving the principal curvatures, depend on six arbitrary constants.

REMARK. Theorem 2 is analogous to S. S. Chern's Theorem for BIisometry [4].

About the third type of BIII-surfaces, we only consider the case of a surface satisfying the equation

$$
P \cos 2 \psi+(Q-1) \sin 2 \psi=0 .
$$

First of all, we get the following. 
Lemma 3. Let $M$ be a BIII-surface, which satisfies (19). Then the following differentials satisfy

$$
d \psi, d P, d Q, d L, d \rho, d a, d c \equiv 0 \quad\left(\bmod \theta_{1}\right) .
$$

Proof. By solving (13), we get

$$
t=(1-Q) P^{-1} \text {. }
$$

Inserting (21) into (12).

$$
P d Q-(Q-1) d P=P(Q-1) \alpha_{1}-P^{2} \alpha_{2}
$$

Taking exterior derivatives of (22), we have

$$
2 d P \wedge d Q=(Q-1) d P \wedge \alpha_{1}+P d Q \wedge \alpha_{1}-2 P d P \wedge \alpha_{2}-P^{2} \alpha_{1} \wedge \alpha_{2}
$$

Taking the wedge product of (22) with $d P, d Q, \alpha_{1}$ and $\alpha_{2}$, respectively we obtain

$(24)_{1-4}$

$$
\begin{gathered}
d P \wedge d Q=(Q-1) d P \wedge \alpha_{1}-P d P \wedge \alpha_{2}, \\
(Q-1) d P \wedge d Q=P(Q-1) d Q \wedge \alpha_{1}-P d Q \wedge \alpha_{2}, \\
P^{2} \alpha_{1} \wedge \alpha_{2}=-(Q-1) d P \wedge \alpha_{1}+P d Q \wedge \alpha_{1}, \\
P(Q-1) \alpha_{1} \wedge \alpha_{2}=-(Q-1) d P \wedge \alpha_{2}+P d Q \wedge \alpha_{2} .
\end{gathered}
$$

Taking derivatives of (19), we get

$$
d P \cos 2 \psi+d Q \sin 2 \psi+2[-P \sin 2 \psi+(Q-1) \cos 2 \psi] d \psi=0 .
$$

From (19),

$$
\cos 2 \psi=\frac{Q-1}{\sqrt{P^{2}+(Q-1)^{2}}}, \quad \sin 2 \psi=\frac{-P}{\sqrt{P^{2}+(Q-1)^{2}}}
$$

Using (19)' and (22), we get

$$
d P \cos 2 \psi+d Q \sin 2 \psi=-P \theta_{1} .
$$

Inserting (26) into (25), we get

$$
2 d \psi=-\theta_{1} \sin 2 \psi
$$

Taking exterior derivatives of (27), we have

$$
\theta_{2} \wedge \theta_{12} \cdot \sin 2 \psi=0 \text {. }
$$

Since $P \neq 0, \sin 2 \psi \neq 0$, and it follows that $\theta_{2} \wedge \theta_{12}=0$, or

$$
\left(P+2 \psi_{1}\right) \cos 2 \psi+\left(Q+2 \psi_{2}\right) \sin 2 \psi=0 .
$$


From (28), using Lemma 2, we have that $M$ is a $W$-surface. Using (28) and (19) we get

$$
2 \psi_{1} \cos 2 \psi+\left(2 \psi_{2}+1\right) \sin 2 \psi=0 .
$$

From (19), (28) and (29),

$$
\operatorname{tg} 2 \psi=\frac{-P}{Q-1}=\frac{-\left(P+2 \psi_{1}\right)}{Q+2 \psi_{2}}=\frac{-2 \psi_{1}}{2 \psi_{2+1}} .
$$

Applying the *-operator to (27), we have

$$
2 * d \psi=-\theta_{2} \sin 2 \psi \text {. }
$$

Taking exterior derivatives of (31), in view of (27), we get

$$
2 d * d \psi=P \alpha_{1} \wedge \alpha_{2} .
$$

On the other hand, from (1.53),

$$
2 d * d \psi=(4 J+P) \alpha_{1} \wedge \alpha_{2} .
$$

By the above two equations, we get $J=0$, or (see (1.46))

$$
P_{1}+Q_{2}+P=0 \text {. }
$$

We denote

$$
d P=P_{i} \alpha_{i}, \quad d Q=Q_{i} \alpha_{i}, \quad i=1,2 .
$$

From (23), (24) $1-4$ and (33), we have

$$
\begin{gathered}
2\left(P_{1} Q_{2}-P_{2} Q_{1}\right)=-(Q-1) P_{2}-P Q_{2}-2 P P_{1}-P^{2}, \\
P_{1} Q_{2}-P_{1} Q_{1}=-(Q-1) P_{2}-P P_{1}, \\
(Q-1)\left(P_{1} Q_{2}-P_{2} Q_{1}\right)=-P\left[(Q-1) Q_{2}+P Q_{1}\right], \\
(Q-1) P_{2}-P Q_{2}=P^{2}, \quad P_{1}+Q_{2}=-P .
\end{gathered}
$$

By solving (35) for $P_{1}, \ldots, Q_{2}$ we get

$$
\frac{P_{2}}{P_{1}}=\frac{Q_{2}}{Q_{1}}=\frac{-P}{Q-1}=\operatorname{tg} 2 \psi
$$

or

$$
\begin{aligned}
& P_{1} \sin 2 \psi-P_{2} \cos 2 \psi=0, \quad Q_{1} \sin 2 \psi-Q_{2} \cos 2 \psi=0, \\
& P P_{1}+(Q-1) P_{2}=0, \quad P Q_{1}+(Q-1) Q_{2}=0 .
\end{aligned}
$$

Using (19), (33) and (1.48), from (1.63), we have

$$
\begin{gathered}
{\left[\frac{1}{2}(\widehat{K}-Q)-L^{-2}\right]\left[\left(P+2 \psi_{1}\right) \cos 2 \psi+\left(Q+2 \psi_{2}\right) \sin 2 \psi\right]} \\
+\frac{1}{4}\left[(\widehat{K}-Q)_{2} \cos 2 \psi-(\widehat{K}-Q)_{1} \sin 2 \psi\right]=0 .
\end{gathered}
$$


Using (28) and (38) implies

$$
(\widehat{K}-Q)_{2} \cos 2 \psi-(\widehat{K}-Q)_{1} \sin 2 \psi=0 .
$$

From (37) and the above equation, we have

$$
\widehat{K}_{2} \cos 2 \psi-\widehat{K}_{1} \sin 2 \psi=0 .
$$

Using (1.49) and its differential, we get

$$
\begin{aligned}
& -\widehat{K}=P^{2}+Q^{2}-P_{2}+Q_{1}, \\
& -\widehat{K}_{1}=2\left(P P_{1}+Q Q_{1}\right)-P_{21}+Q_{11}, \\
& -\widehat{K}_{2}=2\left(P P_{2}+Q Q_{2}\right)-P_{22}+Q_{12} .
\end{aligned}
$$

Using (40), (19)' and (39) implies

$$
\begin{aligned}
& P\left(Q_{11}-P_{21}\right)+(Q-1)\left(Q_{12}-P_{22}\right) \\
& \quad=2\left[P\left(P P_{1}+Q Q_{1}\right)-(Q-1)\left(P P_{2}+Q Q_{2}\right)\right] .
\end{aligned}
$$

Then (11), (19) and (21) imply

$$
t=\operatorname{ctg} \tau=\frac{Q-1}{P}=-\operatorname{ctg} 2 \psi \text {. }
$$

Hence

$$
\tau=-2 \psi+k \pi, \quad k=\text { integer. }
$$

We wish to express the differentials on the left side of (20) in terms of $\theta_{1}$ and $\theta_{2}$. First, from (37) we have

$$
d P=P_{1} \sec 2 \psi \cdot \theta_{1}, \quad d Q=Q_{1} \sec 2 \psi \cdot \theta_{1} .
$$

Furthermore,

$$
\alpha_{12}=P \alpha_{1}+Q \alpha_{2}=\theta_{1}(P \cos 2 \psi+Q \sin 2 \psi)+\theta_{2}(-P \sin 2 \psi+Q \cos 2 \psi) .
$$

From $(19)^{\prime},(27)$, we get

$$
\theta_{12}=\alpha_{12}+2 d \psi=(-P \sin 2 \psi+Q \cos 2 \psi) \theta_{2} .
$$

Using (1.42) gives

$$
d \log L=\left[1+\frac{1}{2}(Q-1) \sec 2 \psi\right] \theta_{1} .
$$

From $(1.50)$,

$$
d \rho=\left[L^{-2}-\frac{1}{2}(\widehat{K}-Q)+\rho(P \sin 2 \psi-Q \cos 2 \psi)\right] \theta_{1} .
$$

Using (27) and (45), from (1.38) it follows that

$$
\omega_{12}=\frac{1}{2} \sin 2 \psi \cdot \theta_{1}-\left\{1+\frac{1}{2}[P \sin 2 \psi-(Q+1) \cos 2 \psi]\right\} \theta_{2} .
$$


From (1.18), (1.19) and (47), we get

$$
d g=f \theta_{1}, \quad d f=f[2 \rho+P \sin 2 \psi-(Q-2) \cos 2 \psi] \theta_{1} .
$$

Using (1.10) and (48), we see that

$$
\begin{aligned}
& 2 d a=f[2 \rho+1+P \sin 2 \psi-(Q-2) \cos 2 \psi] \theta_{1}, \\
& 2 d c=f[-2 \rho+1-P \sin 2 \psi+(Q-2) \cos 2 \psi] \theta_{1} .
\end{aligned}
$$

According to (27), (43), (45), (46) and (49), we obtain (20).

Now we easily obtain the following theorem.

THeOREM 3. Let $M$ be a BIII-surface of the third type, and satisfying equation (19). Then $M$ is a helicoidal surface.

A helicoidal surface in $E^{3}$ is a surface which is invariant under a helicoidal motion:

$$
\begin{aligned}
c_{t}(x) & =x^{\prime}, \quad x=\left(x_{1}, x_{2}, x_{3}\right), \quad x^{\prime}=\left(x_{1}^{\prime}, x_{2}^{\prime}, x_{3}^{\prime}\right), \\
x_{1}^{\prime} & =x_{1} \cos t+x_{2} \sin t, \\
x_{2}^{\prime} & =-x_{1} \sin t+x_{2} \cos t, \quad-\infty<t<+\infty, \\
x_{3}^{\prime} & =x_{3}+b t,
\end{aligned}
$$

where the $x_{3}$-axis is taken as the axis of a helicoidal motion. Let $C$ be a curve parametrized by $s$ :

$$
c(s)=\left(x_{1}(s), x_{2}(s), x_{3}(s)\right) .
$$

Any helicoidal surface $M$ may be considered as the one generated by helicoidal motion of all the points of $C$. Thus its parametrization by $s, t$ is

(50) $x(s, t)=\left(x_{1}(s) \cos t+x_{2}(s) \sin t\right.$,

$$
\left.-x_{1}(s) \sin t+x_{2}(s) \cos t, x_{3}(s)+b t\right),
$$

where $b=$ constant. In other words, on a helicoidal surface there exists a family of helicoidal curves, which have the same helicoidal distance $(b=$ constant) and helicoidal axis.

Proof of Theorem 3. Let us show that on the surface $M$ the set of $\theta_{2}$-curves (the curve along which $\theta_{1}=0$ ) is a family of helicoidal curves.

First of all, from Lemma 3 we conclude that

$$
\psi, P, Q, L, \rho, a, c=\text { constant }\left(\bmod \theta_{1}\right)
$$


that is, they are all constant along the $\theta_{2}$-curves $\left(\theta_{1}=0\right)$. Let us find the curvature $\kappa$ and torsion $\tau$ of the $\theta_{2}$-curves.

According to (1.1) and (1.27), along $\theta_{2}$-curves, we have

$$
\begin{aligned}
& \omega_{1}=a \omega_{13}=-a L^{-1} \theta_{2} \sin \psi, \\
& \omega_{2}=c \omega_{23}=c L^{-1} \theta_{2} \cos \psi .
\end{aligned}
$$

Hence the arc length differential of $\theta_{2}$-curves is

$$
d s=\sqrt{\left(\omega_{1}^{2}+\omega_{2}^{2}\right)}=L^{-1} \sqrt{a^{2} \sin ^{2} \psi+c^{2} \cos ^{2} \psi} \theta_{2} .
$$

Since the angle between tangent directions of $\theta_{2}$-curves and the first principal directions $(\phi=0)$ is $\phi=\psi+\pi / 2$, the normal curvature of $\theta_{2}$-curves, by Euler's theorem is

$$
\begin{aligned}
\kappa_{n} & =a^{-1} \cos ^{2} \phi+c^{-1} \sin ^{2} \phi \\
& =a^{-1} \sin ^{2} \psi+c^{-1} \cos ^{2} \psi .
\end{aligned}
$$

Along $\theta_{2}$-curves, $\theta_{1}=0$ implies $d \psi=0, d \phi=0$. Using (47), we have

$$
\omega_{12}=-\left\{1+\frac{1}{2}[P \sin 2 \psi-(Q+1) \cos 2 \psi]\right\} \theta_{2} .
$$

Using the formula for geodesic curvature $\kappa_{g}=d \phi / d s+\omega_{12} / d s$ and (52) and (54), we obtain the geodesic curvature of a $\theta_{2}$-curve

$$
\kappa_{g}=\frac{-L}{\sqrt{a^{2} \sin ^{2} \psi+c^{2} \cos ^{2} \psi}}\left\{1+\frac{1}{2}[P \sin 2 \psi-(Q+1) \cos 2 \psi]\right\} .
$$

From (51), (53) and (55), $\kappa_{n}$ and $\kappa_{g}$ are constant on each $\theta_{2}$-curve, so its curvature

$$
\kappa=\sqrt{\kappa_{n}^{2}+\kappa_{g}^{2}}=\text { constant. }
$$

Then the torsion of the $\theta_{2}$-curve is given by

$$
\tau=\tau_{g}+d \theta / d s,
$$

where $\tau_{g}$ is the geodesic torsion of the $\theta_{2}$-curve, $\theta$ is the angle between the principal space normal of the $\theta_{2}$-curve and the normal to the surface. We have

(58) $\tau_{g}=\left(c^{-1}-a^{-1}\right) \cos \phi \sin \phi=\left(c^{-1}-a^{-1}\right) \sin \psi \cos \psi, \quad \operatorname{tg} \theta=\kappa_{g} / \kappa_{n}$.

From (51), (57) and (58), torsion $\tau=$ constant along the $\theta_{2}$-curve.

Consequently, we have that the $\theta_{2}$-curves are circular helices which are distinct, in general. Thus the surface $M$ is a helicoidal surface. 


\section{Some special BIII-surfaces}

\section{The minimal BIII-surfaces.}

THEOREM 4. Suppose $M$ and $\stackrel{*}{M}$ are minimal surfaces, and $F: M \rightarrow \stackrel{*}{M}$ is a mapping. Then $F$ is a BI-isometry if and only if $F$ is $a$ BIII-isometry.

Proof. For any surface $M$, we have

$$
\text { III }-2 H \mathrm{II}+K \mathrm{I}=0,
$$

where $H$ and $K$ are the mean curvature and Gaussian curvature, respectively, and I, II and III are the three fundamental forms of $M$. Since $M$ and $\stackrel{*}{M}$ are minimal surfaces, $H=\stackrel{*}{H}=0$ and so $I I I=-K I, ~ I I I I ~=-K^{*}$. When $F$ is a BI or BIII-isometry, $K=\stackrel{*}{K}$. Thus the above equations imply the conclusion of Theorem 4 .

EXAMPLE. A BIII-isometry between the catenoid and the helicoid.

Catenoid $M: m(t, \theta)=(\cosh t \cos \theta, \cosh t \sin \theta, t)$,

Helicoid $\stackrel{*}{M}: \stackrel{*}{m}(u, v)=(u \cos v, u \sin v, v)$,

$$
-\infty<t<\infty, \quad 0 \leq \theta<2 \pi, \quad u \geq 0, \quad 0 \leq v<2 \pi .
$$

The fundamental forms and curvatures of $M$ :

$$
\begin{aligned}
\mathrm{I} & =\cosh ^{2} t\left(d t^{2}+d \theta^{2}\right), \quad H=0, \\
\mathrm{III} & =\cosh ^{-2} t\left(d t^{2}+d \theta^{2}\right), \quad K=-\cosh ^{-4} t .
\end{aligned}
$$

The fundamental forms and curvatures of $\dot{M}$ :

$$
\begin{aligned}
& \quad \stackrel{*}{\mathrm{I}}=d u^{2}+\left(1+u^{2}\right) d v^{2}, \quad \stackrel{*}{H}=0, \\
& \text { III }=\left(1+u^{2}\right)^{-2}\left[d u^{2}+\left(1+u^{2}\right) d v^{2}\right], \quad \stackrel{*}{K}=-\left(1+u^{2}\right)^{-2} .
\end{aligned}
$$

The mapping $F(t, \theta)=(u, v): u=\sinh t, v=\theta$ is both a BI-isometry and BIII-isometry:

$$
\begin{gathered}
F^{*}(\stackrel{*}{\mathbf{I}})=\mathrm{I}, \quad \stackrel{*}{\boldsymbol{F}}(\stackrel{*}{\mathbf{I I I}})=\mathbf{I I I}, \\
\stackrel{*}{H}=H=0, \quad \stackrel{*}{K}=K .
\end{gathered}
$$

\section{The BIII-surfaces of revolution.}

We consider the plane curve $x=\gamma(z)>0, y=0$ and the surface of revolution

$$
M: m(z, \theta)=(\gamma(z) \cos \theta, \gamma(z) \sin \theta, z)
$$


Thus

$$
m_{z}^{\prime}=\left(\gamma^{\prime} \cos \theta, \gamma^{\prime} \sin \theta, 1\right), \quad m_{\theta}^{\prime}=(\gamma \sin \theta, \gamma \cos \theta, 0) .
$$

We choose an orthonormal frame by

$$
\begin{aligned}
& e_{1}=\left(\gamma^{\prime 2}+1\right)^{-1 / 2}\left(\gamma^{\prime} \cos \theta, \gamma^{\prime} \sin \theta, 1\right), \\
& e_{2}=(-\sin \theta, \cos \theta, 0), \\
& e_{3}=e_{1} \times e_{2}=\left(\gamma^{\prime 2}+1\right)^{-1 / 2}\left(-\cos \theta,-\sin \theta, \gamma^{\prime}\right),
\end{aligned}
$$

so that

$$
\begin{aligned}
\omega_{1} & =\left(\gamma^{\prime 2}+1\right)^{-1 / 2} d z, \quad \omega_{2}=\gamma d \theta, \\
\omega_{12} & =h \omega_{1}+k \omega_{2}, \quad h=0, \quad k=\gamma^{\prime}\left(\gamma^{\prime 2}+1\right)^{-1 / 2}, \\
\omega_{13} & =a^{-1} \omega_{1}, \quad a=-\left(\gamma^{\prime \prime}\right)^{-1}\left(\gamma^{\prime 2}=1\right)^{3 / 2}, \quad \gamma^{\prime \prime} \neq 0, \\
\omega_{23} & =c^{-1} \omega_{2}, \quad c=\gamma\left(\gamma^{\prime 2}+1\right)^{1 / 2}, \\
f & =a-c=-\left(\gamma^{\prime \prime}\right)^{-1}\left(\gamma^{\prime 2}+1\right)^{1 / 2}\left(1+\gamma^{\prime 2}+\gamma \gamma^{\prime \prime}\right), \\
g & =a+c=\left(\gamma^{\prime \prime}\right)^{-1}\left(\gamma^{\prime 2}+1\right)^{1 / 2}\left(-1-\gamma^{\prime 2}+\gamma \gamma^{\prime \prime}\right) .
\end{aligned}
$$

THEOREM 5. The surfaces of revolution which are BIII-surfaces are exactly as follows.

(i) Those of the first type $\left(\mathrm{HK}^{-1}=\mathrm{constant}\right)$, which satisfy

$$
\left(\gamma^{\prime 2}+1\right)^{1 / 2}\left(\gamma \gamma^{\prime \prime}-\gamma^{\prime 2}-1\right)=c \gamma^{\prime \prime}, \quad c=\text { constant. }
$$

(ii) Those of the second type, which satisfy

$$
\left[\frac{g^{\prime}\left(\gamma^{\prime 2}+1\right)}{f \gamma^{\prime \prime}}\right]^{\prime}=\left(\frac{g^{\prime}}{f}\right)^{2} \frac{\gamma^{\prime 2}+1}{\gamma^{\prime \prime}} \text {. }
$$

(iii) There are no BIII-surfaces of the third type.

Proof. According to (6) and (1.13), we have

$$
d g=g^{\prime} d z=f\left(u \omega_{13}+v \omega_{23}\right)
$$

It follows that

$$
u=a f^{-1} g^{\prime}\left(\gamma^{\prime 2}+1\right)^{1 / 2}, \quad v=0 .
$$

From (1.16) and (1.17), we get

$$
\begin{aligned}
& \alpha_{1}=\theta_{1}=u \omega_{13}=f^{-1} g^{\prime} d z \\
& \alpha_{2}=\theta_{2}=u \omega_{23}=-\left(f \gamma^{\prime \prime}\right)^{-1} g^{\prime}\left(\gamma^{\prime 2}+1\right) d \theta .
\end{aligned}
$$


Taking exterior derivatives of (10).

$$
d \alpha_{1}=0, \quad d \alpha_{2}=-\left[\frac{g^{\prime}\left(\gamma^{\prime 2}+1\right)}{f \gamma^{\prime \prime}}\right]^{\prime} d z \wedge d \theta .
$$

Using (10), we have

$$
\alpha_{1} \wedge \alpha_{2}=-\left[\frac{g^{\prime}}{f}\right]^{2} \frac{\gamma^{\prime 2}+1}{\gamma^{\prime \prime}} d z \wedge d \theta .
$$

From (11), (12), (1.9) and (1.40), we get

$$
P \equiv 0, \quad Q=\left[\frac{g^{\prime}\left(\gamma^{\prime 2}+1\right)}{f \gamma^{\prime \prime}}\right]^{\prime}\left[\frac{f}{g}\right]^{2} \frac{\gamma^{\prime \prime}}{\gamma^{\prime 2}+1} .
$$

For the first type, from $\alpha_{1}=\alpha_{2}=0$ or $u=v=0$, using (9), $g^{\prime \prime}=0$, $g=c=$ constant, we have that $(6)_{2}$ becomes (7).

For the second type, from $P \equiv 0, Q \equiv 1$, using (13), we have (8).

For the third type, $P \neq 0$, and according to (13) this is not possible. So there are no surfaces in this case.

\section{The BIII-surfaces with constant Gaussian curvature.}

Suppose a surface $M$ has non-zero constant Gaussian curvature $K$ and $H K^{-1} \neq$ constant. Since $d K^{-1}=0$, from (1.19) and (1.21), we get

$$
d f=f\left(\alpha_{1}+2 * \omega_{12}\right)=g \theta_{1}
$$

or

$$
\alpha_{1}+2 * \omega_{12}=\sigma \theta_{1}
$$

where

$$
\sigma=g f^{-1} \neq \pm 1 \text {. }
$$

Note that the inequality in (16) can be concluded from $K \neq 0$. In fact, if $\sigma=(a+c) /(a-c)= \pm 1$, we get $a=0$ or $c=0$, and hence $K=a c=0$. Using (1.2) $)^{\prime}$ and (1.27), rewrite (15) as

$$
1-2 k^{\prime}=\sigma \cos 2 \psi, \quad 2 h^{\prime}=\sigma \sin 2 \psi .
$$

Using (1.38), (1.27) and (15), we get

$$
\theta_{12}=(2 \rho-\sigma) \theta_{2}, \quad * \theta 12=(\sigma-2 \rho) \theta_{1} .
$$

Taking derivatives of (16), using (1.18), and (1.19), we have

$$
d \sigma=\left(1-\sigma^{2}\right) \theta_{1}
$$


According to equation (16), $1-\sigma^{2} \neq 0$, and in view of $(1+\sigma) /(1-\sigma)=$ $(f+g) /(f-g)=-a / c$, from $(19)$ we get

$$
\frac{d \sigma}{1-\sigma^{2}}=\frac{1}{2} d \log \left|\frac{a}{c}\right|=\theta_{1}
$$

Applying the *-operator to (15), we get

$$
\alpha_{2}-2 w_{12}=\sigma \theta_{2} \text {. }
$$

Taking exterior derivatives of (21), from (1.40), (1.5) and (19), we have

$$
(Q-1) / 2=\sigma(1-\sigma)-L^{-2} \text {. }
$$

From (1.50) and (18), we obtain

$$
d \rho=\left[\rho(\sigma-2 \rho)+L^{-2}-\frac{1}{2}(\widehat{K}-Q)\right] \theta_{1}+J \theta_{2} .
$$

From (1.42), we obtain

$$
2 d \log L=(Q-1) \alpha_{1}-P \alpha_{2}+2 \rho \theta_{1} .
$$

Taking derivatives of (22), using (19), (23) and (24), we get

$$
\frac{1}{2} d Q=\lambda \theta_{1}+\mu \theta_{2}+L^{-2}\left[(Q-1) \alpha_{1}-P \alpha_{2}\right],
$$

where

(26) $\lambda=\rho(1-2 \rho \sigma)-2 \sigma\left(1-\sigma^{2}\right)+L^{-2}(\sigma+2 \rho)-\frac{1}{2} \sigma(\widehat{K}-Q), \quad \mu=\sigma J$.

THEOREM 6. There does not exist any BIII-surfaces of the second type such that $K=$ constant $\neq 0, H \neq$ constant .

Proof. For the second type of BIII-surface, we have

$$
P \equiv 0, \quad Q \equiv 1, \quad \widehat{K}=-1 .
$$

Since $K=$ constant $\neq 0, H K^{-1} \neq$ constant, using (27) and (22), we get

$$
L^{-2}=\sigma(1-\sigma) \text {. }
$$

Using (27) and (28), from (23) and (24), we get

$$
\begin{gathered}
d \rho=\left\{1-\left[\rho^{2}+(1-\sigma)^{2}\right]\right\} \theta_{1}, \\
d \log L=\rho \theta_{1} .
\end{gathered}
$$

From (27) it follows that $d Q=0$, and from (25) we have $\lambda=\mu=0$. Using (26), we get $\left(\sigma^{2}-1\right)(\sigma-1)=0$. It follows that $\sigma=1$, in view of $\sigma^{2} \neq 1$. 
From (28) again we have $L^{-2}=0$, contradicting that $H K^{-1} \neq$ constant, $L \neq 0$. So the surface cannot exist.

\section{Acknowledgement}

The author is grateful to Professor Hyam Rubinstein for his help and suggestions, and to Professor S. S. Chern for his suggestions and encouragement.

\section{References}

[1] O. Bonnet, 'Memoire sur la theorie des surfaces applicables', J. Ecole Polytechnique 42 (1867), 72-92.

[2] E. Cartan, 'Couples des surfaces applicables avec conservation des courbures principales', Bull. Sci. Math. 66 (1942), 55-85.

[3] S. S. Chern, 'Deformation of surfaces preserving principal curvatures', Differential geometry and complex analysis, Springer Verlag, New York, 1985, pp. 155-163.

[4] W. C. Graustein, 'Applicability with preservation of both curvatures', Bull. Amer. Math. Soc. 30 (1924), 19-27.

[5] I. M. Roussos, 'Mean-curvature-preserving isometries of surfaces in ordinary space', to appear.

[6] Wenmao Yang, 'Infinitesimal $O$. Bonnet-deformations of surfaces in $E^{3}$, Differential Geometry and Topology, Proceedings, Tianjin 1986-87, edited by Boju Jiang, Chia-Kuei Peng and Zixin Hou, pp. 306-321 (Lecture Notes in Mathematics, no. 1369, Springer Verlag, New York, 1989).

Wuhan University

Wuhan, Hubei

People's Republic of China 